\title{
Optimal Spatial Filtering of Single Trial EEG During Imagined Hand Movement
}

\author{
Herbert Ramoser, Johannes Müller-Gerking, and Gert Pfurtscheller
}

\begin{abstract}
The development of an electroencephalograph (EEG)-based brain-computer interface (BCI) requires rapid and reliable discrimination of EEG patterns, e.g., associated with imaginary movement. One-sided hand movement imagination results in EEG changes located at contra- and ipsilateral central areas. We demonstrate that spatial filters for multichannel EEG effectively extract discriminatory information from two populations of single-trial EEG, recorded during left- and right-hand movement imagery. The best classification results for three subjects are $90.8 \%, 92.7 \%$, and $99.7 \%$. The spatial filters are estimated from a set of data by the method of common spatial patterns and reflect the specific activation of cortical areas. The method performs a weighting of the electrodes according to their importance for the classification task. The high recognition rates and computational simplicity make it a promising method for an EEG-based brain-computer interface.
\end{abstract}

Index Terms-Assistive communication, electroencephalograph (EEG) classification, event-related desynchronization (ERD), mu rhythm, prosthesis, sensorimotor cortex.

\section{INTRODUCTION}

$\mathbf{P}$ ATIENTS in a late stage of amyotropic lateral sclerosis (ALS) or suffering from a locked-in syndrome are not able to produce any voluntary muscle movements. Sensory and cognitive functions of the brain are only minimally affected by such a disease. One possibility to open a communication channel for these patients is to use electroencephalograph (EEG) signals to control an assistive device that allows, for example, the selection of letters on a screen [brain-computer interface (BCI)]. It has been shown that the imagination of a limb activation can modify brain electrical activity [1]. Depending on the type of motor imagery, different EEG patterns can be obtained. Activation of hand area neurons either by preparation for a real movement or by imagination of the movement is accompanied by an circumscribed event-related desynchronization (ERD) [2] focused at the hand area [3].

In a number of experiments, we found that EEG signals recorded from two bipolar channels over the left- and right-hand areas during imagined one-sided hand movements could be

Manuscript received August 10, 1998; revised March 22, 1999. This work was supported by the Austrian Fonds zur Förderung der wissenschaftlichen Forschung under Project P11208MED.

H. Ramoser is with the Department of Medical Informatics, Institute of Biomedical Engineering, Graz University of Technology, Graz A-8010, Austria (e-mail: ramoser@dpmi.tu-graz.ac.at).

J. Müller-Gerking is with HLRZ, Forschungszentrum, Jülich D-52425 Germany.

G. Pfurtscheller is with the Ludwig Boltzmann Institute of Medical Informatics and Neuroinformatics, Graz University of Technology, Graz 8010, Austria (e-mail: pfu@dpmi.tu-graz.ac.at).

Publisher Item Identifier S 1063-6528(00)09838-4.

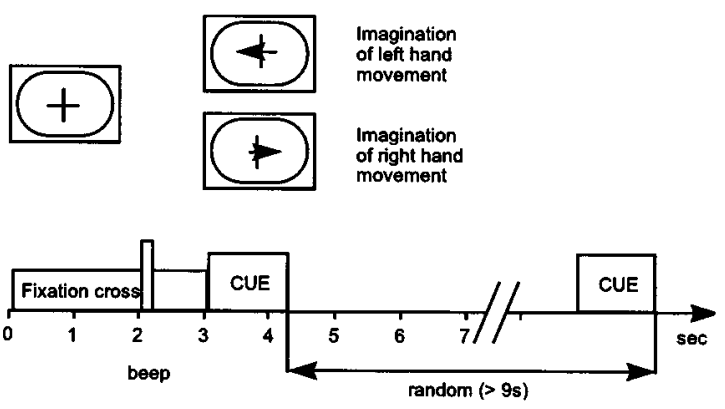

Fig. 1. Timing of the movement imagery task. The cue stimulus in form of an arrow gives the side of imagination.

differentiated with an accuracy of about 85\% [3] (accuracy refers to the number of correctly classified trials divided by the overall number of trials). This accuracy compares quite well to other studies [4] but it is still too low for a BCI. In current prototypes of a BCI, patients with ALS need about one minute to select a letter, when the single trial accuracy is $80 \%$ [17]. If we could achieve a classification accuracy close to $100 \%$, the required time for selection of a letter would be dramatically reduced, since fewer corrections are necessary after choosing a wrong letter.

There are different ways to increase the classification accuracy of an EEG-based BCI.

1) In the case when only one or two EEG channels are available, more advanced algorithms for the extraction of discrimination-relevant features might be used (e.g., adaptive autoregressive filtering [5]) and/or better classifiers might be employed [e.g., finite impulse response (FIR) multilayer perceptrons] [6], [7].

2) The use of multiple EEG channels to acquire additional information about the state of the brain.

Recently, an analysis of multichannel EEG data has shown that classification accuracy can be increased by training an artificial neural network for each EEG channel and combining all networks to a committee [8]. A different approach used specifically designed spatial filters obtained by the method of common spatial patterns (CSP) to construct very few new time-series whose variances contained the most discriminative information. These were classified by a linear discriminator [9].

The method of common spatial patterns was first applied to EEG for detection of abnormalities [10] and later used to discriminate movement-related patterns [9]. The method of CSP is based on a decomposition of the raw EEG signals into spatial patterns, which are extracted from two populations of single trial EEG. These patterns maximize the difference between the populations. In this study, one population consists of EEG recordings 
during left motor imagery and the other population consists of right motor imagery data.

The goal of this paper is to apply the method of CSP to 56-channel EEG recordings obtained during right and left motor imagery and to investigate whether multiple channels can improve the classification accuracy as compared to two channels. The CSP method is applied to common reference EEG data and to computed reference-free data.

\section{METHODS}

\section{A. Experiment and Data Acquisition}

1) Subjects: Three female right-handed subjects (age 20-27 years) took part in the study. The student volunteers were paid for their participation.

2) Procedure: The subjects were seated in an armchair and looked at a computer monitor placed approximately $2 \mathrm{~m}$ in front at eye level. They were asked to keep their arms and hands relaxed and to avoid eye movements during the recordings. Each trial started with the presentation of a fixation cross at the center of the monitor, followed by a short warning tone ("beep") at $2 \mathrm{~s}$ (Fig. 1). At $3 \mathrm{~s}$, the fixation cross was overlaid with an arrow at the center of the monitor for $1.25 \mathrm{~s}$, pointing either to the right or to the left ("cue"). Depending on the direction of the arrow, the subject was instructed to imagine a movement of the right or the left hand. The sequence of right and left trials, as well as the duration of the breaks between consecutive trials (ranging between 0.5 and $2.5 \mathrm{~s}$ ), was randomized. Thus, the interval between consecutive cue stimuli was at least $9 \mathrm{~s}$. The experiment comprised four experimental runs of 40 trials each (20 left and 20 right trials).

3) Recordings: EEG was recorded referentially from 56 $\mathrm{Ag} / \mathrm{AgCl}$ electrodes placed over central and related areas, equally spaced with approximately $2.5 \mathrm{~cm}$ distance. The reference electrode was mounted on the right ear and the grounding electrode on the forehead. The EEG was filtered in a $0.5-50$ $\mathrm{Hz}$ frequency band. Electrooculogram (EOG) was derived bipolarly using two electrodes, one placed medially above and the other laterally below the right eye. In addition, the surface electromyogram (EMG) was obtained from the $m$ extensor digitorum communis of the right and left forearm to detect task-related muscle activity. The EMG was filtered in a 1.5-1 $\mathrm{kHz}$ frequency range and rectified. A contour follower with a 4-Hz upper frequency limit was used to extract the overall EMG activity. All signals, including 56 EEG signals, EOG, and the EMG contour, were sampled at $128 \mathrm{~Hz}$.

\section{B. Data Preprocessing}

1) Artifact Detection: All trials were visually checked for EEG artifacts during the movement imagery period (i.e., second $4-8$ ). From the total of 160 trials recorded, all trials containing artifacts found during visual inspection, e.g., task-related EMG (i.e., hand movements during the imagination period) or EOG activity were omitted from further analysis, leaving 149 (74 left, 75 right), 142 (75/67), and 115 (59/56) trials for subjects S1, S2, and $\mathrm{S} 3$, respectively.
2) EEG Rereferencing: The selection of a suitable EEG reference can greatly influence the classification accuracy and sensitivity to artifacts [11], [12]. In this study we use referential, small Laplacian, large Laplacian, bipolar, and common average referenced (CAR) data.

A small (large) Laplacian reference is obtained by rereferencing an electrode to the mean of its four nearest (next-nearest) neighboring electrodes. For bipolar recordings, the difference of the electrodes in anterior and posterior direction gives the rereferenced signal. A common average reference uses the mean of all electrodes as reference [13].

For bipolar, small, and large Laplacian rereferencing, border electrodes are omitted; hence, 34, 30, and seven EEG channels remain for these methods.

3) Filters: Prior to calculation of the spatial filters, the rereferenced EEG signal was filtered in an $8-30 \mathrm{~Hz}$ band. The filter used was a zero-phase forward/backward FIR filter with a width of 20 points. The frequency band was chosen because it encompasses the alpha and beta frequency bands, which have been shown to be most important for movement classification [3]. Furthermore, in a recent movement study, it was shown that a broad frequency band (e.g., 8-30 Hz) gives better classification results compared to narrow bands [9].

\section{Spatial Filters}

The goal of this study is to design spatial filters that lead to new time series whose variances are optimal for the discrimination of two populations of EEG related to left and right motor imagery. The method used to design such spatial filters is based on the simultaneous diagonalization of two covariance matrices [14]. This method, called the method of common spatial patterns, has been introduced to EEG analysis for detection of abnormal EEG [10] and was recently applied successfully to the classification of movement-related EEG [9].

For the analysis, the raw EEG data of a single trial is represented as an $N \times T$ matrix $E$, where $N$ is the number of channels (i.e., recording electrodes) and $T$ is the number of samples per channel. The normalized spatial covariance of the EEG can be obtained from

$$
C=\frac{E E^{\prime}}{\operatorname{trace}\left(E E^{\prime}\right)}
$$

where' denotes the transpose operator and trace $(x)$ is the sum of the diagonal elements of $x$. For each of the two distributions to be separated (i.e., left and right motor imagery), the spatial covariance $\bar{C}_{d}, \in[l, r]$ is calculated by averaging over the trials of each group. The composite spatial covariance is given as

$$
C_{c}=\bar{C}_{1}+\bar{C}_{r}
$$

$C_{c}$ can be factored as $C_{c}=U_{c} \lambda_{c} U_{c}^{\prime}$, where $U_{c}$ is the matrix of eigenvectors and $\lambda_{c}$ is the diagonal matrix of eigenvalues. Note that throughout this section, the eigenvalues are assumed to be sorted in descending order.

The whitening transformation

$$
P=\sqrt{\lambda_{c}^{-1}} U_{c}^{\prime}
$$


equalizes the variances in the space spanned by $U_{c}$, i.e., all eigenvalues of $P C_{c} P^{\prime}$ are equal to one. If $\bar{C}_{1}$ and $\bar{C}_{r}$ are transformed as

$$
S_{1}=P \bar{C}_{1} P^{\prime} \quad \text { and } \quad S_{r}=P \bar{C}_{r} P^{\prime}
$$

then $S_{1}$ and $S_{r}$ share common eigenvectors, i.e.,

$$
\text { if } S_{1}=B \lambda_{1} B^{\prime} \quad \text { then } \quad S_{r}=B \lambda_{r} B^{\prime} \quad \text { and } \quad \lambda_{1}+\lambda_{r}=I
$$

where $I$ is the identity matrix. Since the sum of two corresponding eigenvalues is always one, the eigenvector with largest eigenvalue for $\bar{S}_{1}$ has the smallest eigenvalue for $\bar{S}_{r}$ and vice versa. This property makes the eigenvectors $B$ useful for classification of the two distributions. The projection of whitened EEG onto the first and last eigenvectors in $B$ (i.e., the eigenvectors corresponding to the largest $\lambda_{1}$ and $\lambda_{r}$ ) will give feature vectors that are optimal for discriminating two populations of EEG in the least squares sense.

With the projection matrix $W=\left(B^{\prime} P\right)^{\prime}$, the decomposition (mapping) of a trial $E$ is given as

$$
Z=W E \text {. }
$$

The columns of $W^{-1}$ are the common spatial patterns and can be seen as time-invariant EEG source distribution vectors.

\section{Classification}

The features used for classification are obtained by decomposing (filtering) the EEG according to (6). For each direction of imagined movement, the variances of only a small number $m$ of signals most suitable for discrimination are used for the construction of the classifier. The signals $Z_{p}(p=1 \cdots 2 m)$ that maximize the difference of variance of left versus right motor imagery EEG are the ones that are associated with the largest eigenvalues $\lambda_{1}$ and $\lambda_{r}$. These signals are the $m$ first and last rows of $Z$ due to the calculation of $W$

$$
f_{p}=\log \left(\frac{\operatorname{var}\left(Z_{p}\right)}{\sum_{i=1}^{2 m} \operatorname{var}\left(Z_{i}\right)}\right) \text {. }
$$

The feature vectors $f_{p}$ of left and right trials are used to calculate a linear classifier [15]. The log-transformation serves to approximate normal distribution of the data.

For proper estimation of the classification accuracy, the data set of each subject is divided into a training and testing set. The training set is used to calculate a classifier, which is used to classify the testing set. This training/testing procedure is repeated 20 times with different random partitions into training and testing sets (i.e., $20 \times$ cross-validation).

\section{RESULTS}

For calculation of the spatial filters, each trial is split into nonoverlapping time segments of $1.5 \mathrm{~s}$ length (i.e., $T=192$ in the equations above). For each of these segments, spatial filters and classifiers are calculated using the formulas in the preceding section, and the classification accuracy is determined by
TABLE I

Classification ACCURACY FOR EACH

SubJeCt, Five EEG REFERENCING METHOdS, AND VARYING Numbers of Channels. Only the Classification Accuracies of the Single Best Time Segment of Each Subject are Given. the Best Result of EACH SubJeCt Is Set In Bold FACE

\begin{tabular}{lc|ccc}
\hline & & S1 & S2 & S3 \\
Derivation & Channels & Acc. [\%] & Acc. [\%] & Acc. [\%] \\
\hline referential & 56 & $92.1 \pm 3.3$ & $89.0 \pm 4.0$ & $\mathbf{9 9 . 7 \pm 1 . 5}$ \\
referential & 18 & $91.0 \pm 3.3$ & $90.4 \pm 3.6$ & $95.0 \pm 8.3$ \\
referential & 2 & $78.8 \pm 4.9$ & $80.4 \pm 4.6$ & $65.0 \pm 10.3$ \\
bipolar & 34 & $\mathbf{9 2 . 7} \pm 2.8$ & $88.2 \pm 4.7$ & $97.7 \pm 3.9$ \\
small Lap. & 30 & $92.3 \pm 3.1$ & $89.9 \pm 4.4$ & $98.0 \pm 3.1$ \\
large Lap. & 7 & $91.1 \pm 3.0$ & $88.4 \pm 5.0$ & $95.0 \pm 4.8$ \\
large Lap. & 2 & $87.4 \pm 3.9$ & $78.6 \pm 4.1$ & $93.0 \pm 6.3$ \\
CAR & 56 & $91.5 \pm 3.8$ & $89.4 \pm 4.3$ & $98.3 \pm 3.7$ \\
CAR & 18 & $90.8 \pm 3.4$ & $\mathbf{9 0 . 8} \pm 4.0$ & $95.0 \pm 6.1$
\end{tabular}

the cross-validation procedure. For each direction, only the two most important filters (i.e., $m=2$ in the equations above) are used for setting up a classifier. It has been shown that the use of more filters does not significantly improve classification accuracy [9].

In the remainder of this section, we consider only the time segment with the highest classification accuracy. For subjects S1 and $\mathrm{S} 2$, all methods give the best results for the segment 4.5-6 s; for subject $\mathrm{S} 3$, the segment $3-4.5 \mathrm{~s}$ is selected.

\section{A. Accuracy}

Table I shows the classification accuracy for each subject and each EEG referencing method. For three of the referencing methods, results for electrode subsets are included. These electrode subsets are 18 electrodes surrounding $\mathrm{C} 3$ and $\mathrm{C} 4$. The results for two EEG channels were obtained by designing a linear classifier for the variances of the rereferenced EEG signals of electrodes $\mathrm{C} 3$ and $\mathrm{C} 4$, i.e., without application of the CSP method.

For each of the subjects, an accuracy of more than $90 \%$ can be achieved. For subject S3, several methods give results close to $100 \%$. The referencing method has little influence on the classification accuracy. Taking more than two channels sensibly increases performance. For example, a referential recording using 18 channels increases the performance by more than $10 \%$ compared to two channels. A further increase to 56 channels does not significantly improve performance. For a large Laplacian reference, the results are less dependent on the number of channels. It must, however, be kept in mind that the large Laplacian reference with two channels actually requires a montage with ten electrodes.

\section{B. Spatial Patterns}

Fig. 2 shows the spatial patterns calculated for subject S1 [i.e., the first and last columns of $W^{-1}$ in (6)]. The contour plots are obtained with cubic interpolation. Since within a pattern the coefficients seldomly cross the zero line, and for filtering only the absolute value of the coefficients is of importance, the patterns 
are plotted in grayscale symmetric to zero to allow for easier comparison.

Left motor imagery causes a relatively increased EEG variance over the left hemisphere because on the contralateral hemisphere, event-related desynchronization of EEG takes place. This behavior is reflected in large coefficients for electrodes on the left hemisphere in the most important spatial pattern. Mirrored behavior can be seen for right motor imagery.

The most important patterns show their strongest modulation at electrodes above the motor cortex. The pattern for left motor imagery is focused at electrode $\mathrm{C} 3$ overlaying the hand area. However, the focus for right imagery is at electrodes slightly anterior to $\mathrm{C} 4$. The second most important patterns do not exhibit a clear focus at specific electrodes.

\section{Subjects}

Figs. 3-5 display the most important spatial patterns for all subjects with referential, small Laplacian, and bipolar references.

The patterns for all referencing methods are very similar. Because of the spatial filtering properties of the small Laplacian reference (actually, this reference approximates a spatial second derivative), it shows more distinct spatial differences.

For each subject, the focus of the patterns is at the same electrodes regardless of the referencing method, and, generally, electrodes overlaying the hemisphere opposite to the focus have coefficients close to zero. For none of the subjects are the patterns for left and right imagery are centered exactly at $\mathrm{C} 3$ and $\mathrm{C} 4$, which are currently used for EEG classification in the BCI project [3]. Especially in the right hemisphere, the electrode with maximum discriminatory power is anterior to $\mathrm{C} 4$ for subject $\mathrm{S} 1$ and posterior to $\mathrm{C} 4$ for subjects $\mathrm{S} 2$ and $\mathrm{S} 3$. This displacement of the focus relative to electrodes $\mathrm{C} 3$ and $\mathrm{C} 4$ partly explains the increase in classification accuracy with multiple channels with respect to a two-channel montage.

\section{DISCUSSION}

All three subjects have participated in a series of motor imagery sessions (left versus right hand) with delayed feedback and two bipolar EEG channels prior to the reported multichannel experiment [3]. The best classification results in these sessions were $91.3 \%$ for subject S1, $83.7 \%$ for S2, and $89.9 \%$ for S3. These results were obtained online in a feedback session whereby in each subject, the bandpower of two optimized narrow frequency bands was classified. The use of the common spatial patterns method increases the classification accuracy between 1\% (subject S1) and 10\% (subject S3), even though in the multichannel experiment no feedback was given. From the inspection of the ERD time course calculated for electrodes $\mathrm{C} 3$ and $\mathrm{C} 4$ during left and right motor imagery in experiments with and without feedback, it can be expected that in the latter case the classification accuracy is lower [3, Fig. 3].

Imagination of left (right) movement leads to reduced amplitude of sensorimotor rhythms over the right (left) hemisphere. The topographical display of the ERD during motor imagery in subjects S1, S2, and S3 showed a focal ERD over the contralateral primary sensorimotor area. Additionally, in two of the
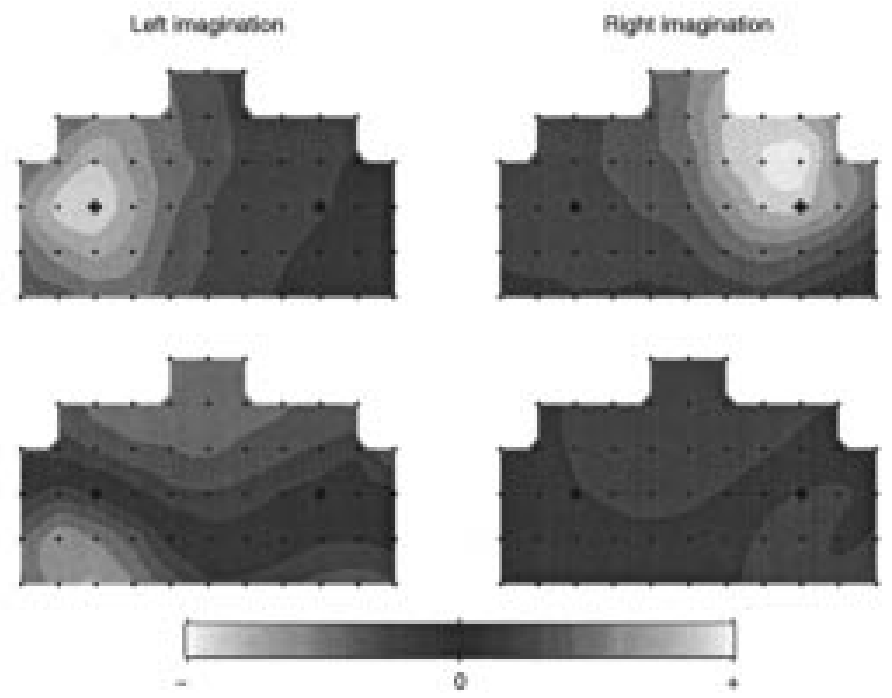

Fig. 2. Most important and second most important spatial patterns for the discrimination of left from right motor imagery. Subject S1, referential recording, 56 channels. Electrode positions are marked with a black dot except for electrodes C3 (left) and C4 (right), which are marked with a "+." All electrodes are arranged in a grid of $2.5-\mathrm{cm}$ spacing.
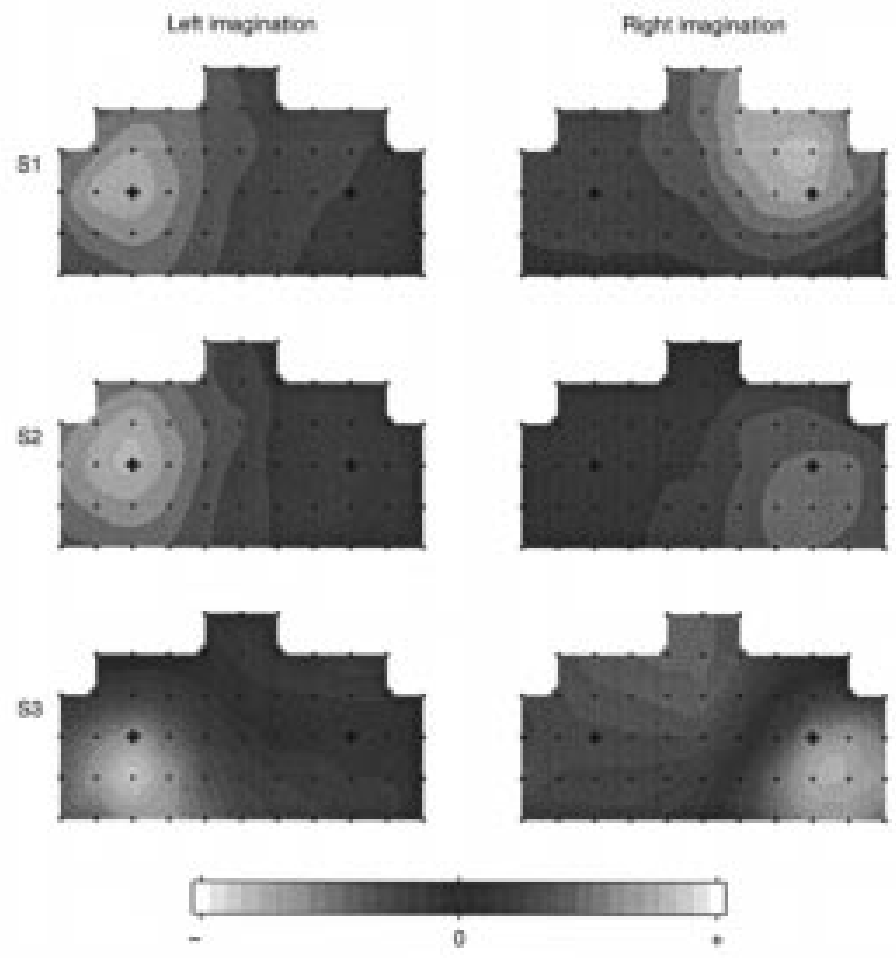

Fig. 3. Most important spatial patterns for the detection of left and right motor imagery for each subject and referential recording, 56 channels. See Fig. 2 for further explanation.

subjects (S2 and S3), ipsilateral event-related synchronization (ERS) was present [16, Fig. 2]. It is therefore not surprising that the relative increase of EEG variance as indicated in the spatial patterns is focused on the ipsilateral side.

In online experiments with two bipolar EEG channels, the most reactive frequency bands were $9-13 \mathrm{~Hz}$ for subject $\mathrm{S} 1$, 10-12 $\mathrm{Hz}$ for S2, and 22-29 Hz for S3 [16]. In these bands, the EEG reactivity (i.e., ERD and ERS) was most impressive and 


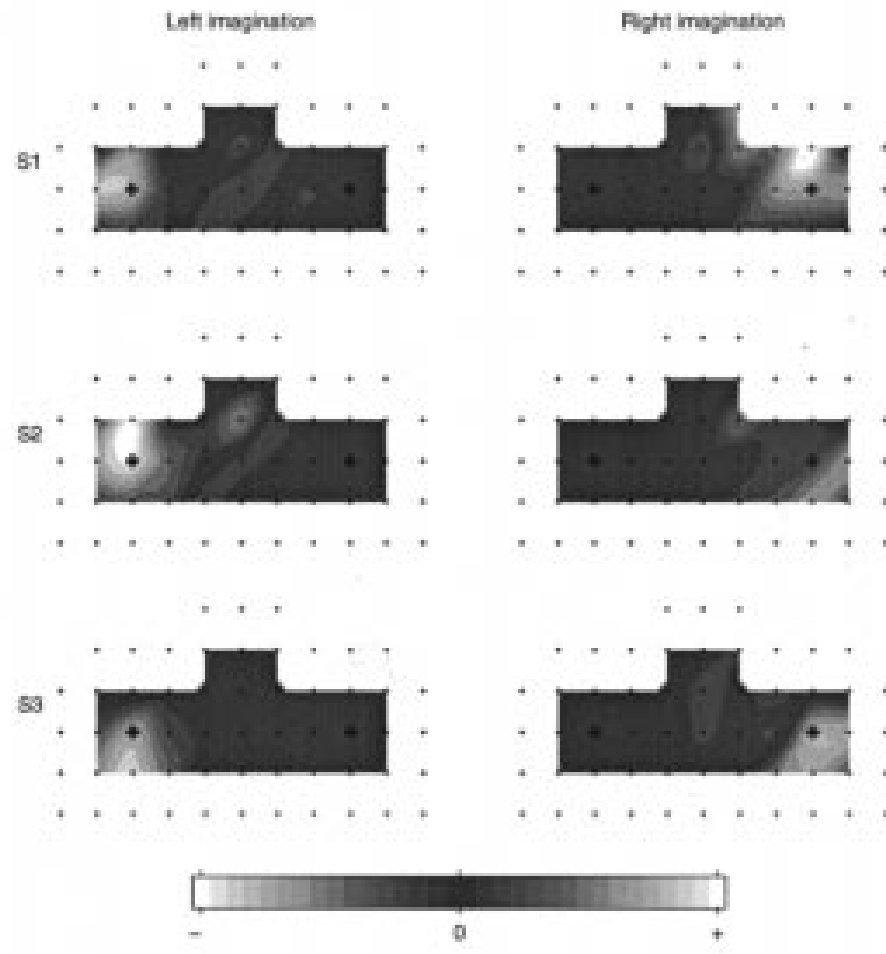

Fig. 4. Most important spatial patterns for the detection of left and right motor imagery for each subject and small Laplacian reference, 30 channels. See Fig. 2 for further explanation.

showed largest differences between both imagination tasks. The analyzed frequency band of $8-30 \mathrm{~Hz}$ covers all of these most reactive frequency components. It is of interest that especially in subject S3 with a reactive beta frequency band, the classification results with the CSP method are close to $100 \%$. In this subject, classification of two channels (large Laplacian reference) results already in an accuracy of $93 \%$. This can be interpreted that for subject S3, the most relevant information for differentiation of left and right motor imagery is in the central beta rhythm located at the Rolandic region. In the other subjects, a more widespread activity in the alpha band explains the relatively poor classification results with two channels and the improvement with additional channels.

The major problem in the application of the method presented in this paper is the sensitivity to artifacts in the EEG. In the reported study, we use the sample covariance to estimate the covariance matrices, which are the basis for calculation of the spatial filters. This estimator is known to be nonrobust. Since the covariance matrices are estimated with a comparatively small number of examples (e.g., 100 trials with 192 samples each for a $56 \times 56$ covariance matrix), a single trial contaminated with artifacts can cause severe changes to the filters. Therefore, the method currently requires EEG data free of any artifact for reliable calculation of the spatial filters. The use of a robust variance estimation method may circumvent these problems. Once the filters have been calculated, the influence of artifacts is reduced since each filter performs weighted spatial averaging of the EEG. Further possible drawbacks of the CSP method are as follows.

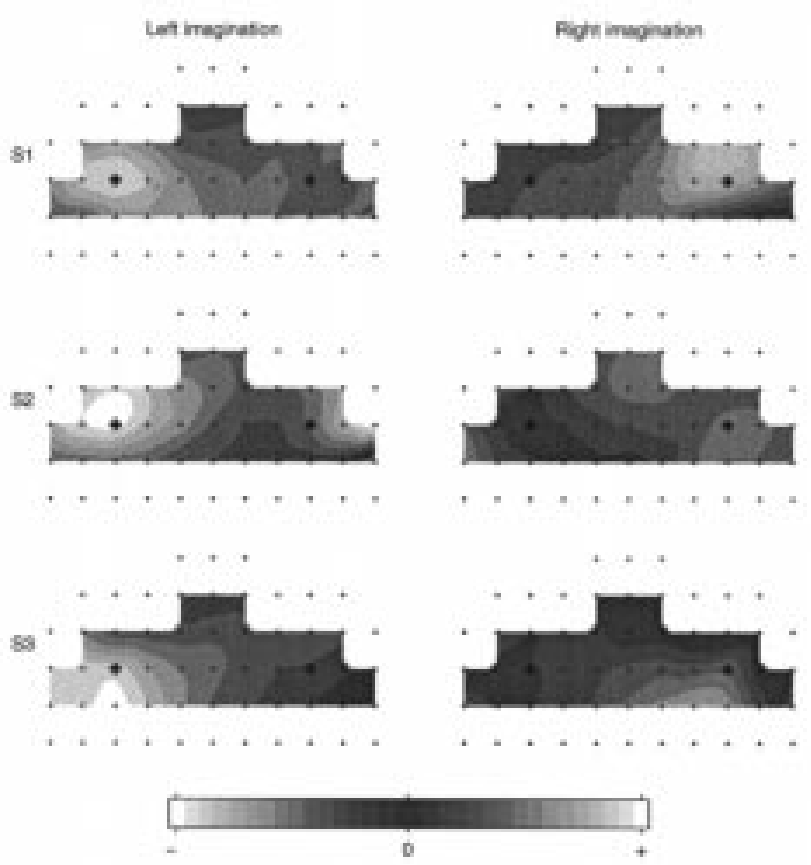

Fig. 5. Most important spatial patterns for the detection of left and right motor imagery for each subject and bipolar reference, 34 channels. See Fig. 2 for further explanation.

1) The need for at least 18 EEG electrodes, which necessitates costly hardware and also makes electrode and hardware failures more likely.

2) The CSP method detects spatial patterns in the EEG.

Hence, changing electrode positions may render the improvements in classification accuracy gained by the method useless. The method, therefore, requires almost identical electrode positions for all trials and sessions, which is difficult to accomplish.

The EEG referencing method has little influence on the classification accuracy. However, another study indicates that large Laplacian and CAR references are most suited for a BCI [11]. These contradictory results may be caused by the fact that the data in this study were free of artifacts, and therefore, the rereferencing methods could not improve the signal-to-noise ratio. A noise-insenitive rereferencing method, e.g., a small Laplacian, may prove useful when the data are not completely free of artifacts.

The results also indicate that 18 channels covering the hand areas of the motor cortex are sufficient for good classification. A further increase in the number of channels does not significantly improve accuracy. The use of two EEG channels with a large Laplacian reference gives satisfactory classification accuracy for two of the subjects. Hhowever, this referencing method requires a total of ten EEG electrodes.

The methods used in this study do not take into account temporal information of the filtered EEG signal. Solely, the variance of the spatially filtered EEG is used for classification. The use of time-dependent parameters estimated from the spatially filtered EEG (e.g., adaptive autoregressive models) may further improve classification accuracy. Results of a similar study indicate that the use of more advanced (nonlinear) methods for classification of the variances does not improve classification accuracy [9]. 


\section{REFERENCES}

[1] S. C. Gandevia and J. C. Rothwell, "Knowledge of motor commands and the recruitment of human motoneurons," Brain, vol. 110, no. 5, pp. 1117-1130, 1987.

[2] G. Pfurtscheller, "Graphical display and statistical evaluation of eventrelated desynchronization (ERD)," Electroenc. Clin. Neurophys., vol. 43, pp. 757-760, 1977.

[3] G. Pfurtscheller, C. Neuper, D. Flotzinger, and M. Pregenzer, "EEGbased discrimination between imagination of right and left hand movement," Electroenc. Clin. Neurophys., vol. 103, no. 5, pp. 1-10, 1997.

[4] J. R. Wolpaw, D. J. McFarland, G. W. Neat, and C. A. Forneris, "An EEG-based brain-computer interface for cursor control," Electroenc. Clin. Neurophys., vol. 78, pp. 252-259, 1991.

[5] G. Pfurtscheller, C. Neuper, A. Schloegl, and K. Lugger, "Separability of EEG signals recorded during right and left motor imagery using adaptive autoregressive parameters," IEEE Trans. Rehab. Eng., vol. 6, pp. 316-325, Sept. 1998.

[6] E. Haselsteiner and G. Pfurtscheller, "Using time dependent neural networks for EEG classification," IEEE Trans. Rehab. Eng., vol. 6, pp. 457-463, Dec. 2000.

[7] S. Haykin, Neural Networks: A Comprehensive Foundation. New York: Macmillan, 1994.

[8] B. O. Peters, G. Pfurtscheller, and H. Flyvbjerg, "Prompt recognition of brain states by their EEG signals," Theory Biosci., vol. 116, pp. 290-301, 1997.

[9] J. Müller-Gerking, G. Pfurtscheller, and H. Flyvbjerg, "Designing optimal spatial filters for single-trial EEG classification in a movement task," Electroenc. Clin. Neurophys., 1999, to be published.

[10] Z. J. Koles, "The quantitative extraction and topographic mapping of the abnormal components in the clinical EEG," Electroenc. Clin. Neurophys., vol. 79, pp. 440-447, 1991.

[11] D. J. McFarland, L. M. McCane, S. V. David, and J. R. Wolpaw, "Spatial filter selection for EEG-based communication," Electroenc. Clin. Neurophys., vol. 103, no. 3, pp. 386-394, 1997.

[12] G. Pfurtscheller, "Mapping of event-related desynchronization and type of derivation," Electroenc. Clin. Neurophys., vol. 70, pp. 190-193, 1988.

[13] — " "Mapping procedures," in Digital Biosignal Processing, R. Weitkunat, Ed: Elsevier, 1991, vol. 5, Techniques in Behavioral and Neural Sciences.

[14] K. Fukunaga, Introduction to Statistical Pattern Recognition, 2nd ed. New York: Academic , 1990
[15] R. O. Duda and P. E. Hart, Pattern Classification and Scene Analysis. New York: Wiley, 1973

[16] G. Pfurtscheller and C. Neuper, "Motor imagery activates primary sensorimotor areas," Neurosci. Lett., vol. 239, pp. 65-68, 1997.

[17] private communication.

Herbert Ramoser received the M.Sc. degree in telematics from the Graz University of Technology, Austria, in 1997, where he is currently pursuing the Ph.D. degree.

He is currently a Research Associate with the Graz University of Technology. His primary research interests are blind separation techniques, signal processing, and application of neural networks. Currently, his research focuses on spatial properties of EEG and sleep analysis

Johannes Müller-Gerking received the master's degree in physics from the University of Constance, Germany, in 1990 and the Ph.D. degree in cognitive sciences from the Université de Paris VI, France, in 1995.

From 1991 to 1994, he was a Fellow of the Gottlieb Daimler- and Karl BenzStiftung, Germany. Until 1996, he was a Research Assistant at NSMA, University of Arizona. He was a Member of the Von Neuman Institute for Computing at the Research Center Jülich, Germany. His area of expertise is linear and nonlinear time series analysis of electrophysiological signals.

Gert Pfurtscheller received the M.Sc. and Ph.D. degrees in electrical engineering from Graz University of Technology, Austria, in 1964 and 1966, respectively.

He is a Professor of medical informatics at the Institute of Biomedical Engineering, Graz University of Technology. Since 1982, he has been Head of the Department of Medical Informatics, and since 1987 Director of the Ludwig Boltzmann-Institute for Medical Informatics and Neuroinformatics. He has authored more than 300 scientific articles and four books. His scientific interests and current research work include biomedical signal processing with special emphasis on electrical brain activity analysis, brain-computer communication systems, and medical expert systems. 